\title{
Measuring the benefits of growth hormone therapy in children: a role for preference-based approaches?
}

\author{
Stavros Petrou, ${ }^{1,2}$ Emma McIntosh ${ }^{1}$
}

Recombinant human growth hormone has revolutionised the management of children with growth hormone deficiency, chronic renal insufficiency, Turner syndrome, Prader-Willi syndrome, small-forgestational-age status, idiopathic short stature and other growth disorders in recent decades. The benefits of administering growth hormone to children have commonly been measured in terms of acceleration in linear growth. ${ }^{1}$ Although informative, lines of enquiry that measure the benefits of recombinant human growth hormone in natural or physical units, such as acceleration in linear growth, suffer from a number of limitations. Most notably, they are unable to incorporate the several health changes that can result from growth hormone therapy, including the increased risk of a number of adverse effects ${ }^{1}$ such as pigmentation and growth of nevi, gynecomastia, pancreatitis and benign intracranial hypertension, within a single measure. In addition, they overlook the broader effects that the treatment may have on children's physical, mental and social well-being, as well individual preferences for those health changes. A number of preference-based approaches for measuring the benefits of healthcare interventions have been developed by health economists and might usefully be applied to growth hormone therapy in children. One such approach is conjoint analysis, the application of which is illustrated by Ahmed et $a l^{2}$ in this issue of the journal in relation to preferences for growth hormone injection devices. This is contrasted with the study by Kapoor et $a l^{3}$ also published in this issue of the journal,

\footnotetext{
${ }^{1}$ Health Economics Research Centre, Department of Public Health, University of Oxford, Oxford, UK; ${ }^{2}$ National Perinatal Epidemiology Unit, Department of Public Health, University of Oxford, Oxford, UK

Correspondence to: Dr S Petrou, National Perinatal Epidemiology Unit, University of Oxford (Old Road Campus), Old Road, Headington, Oxford OX3 7LF, UK; stavros.petrou@npeu.ox.ac.uk
}

which measures the impact of growth hormone-prescribing policies on non-preference-based outcomes, namely treatment concordance and height velocity.

Preference-based approaches that can be used to measure the benefits of growth hormone therapy in children include quality adjusted life year (OALY)-based approaches, contingent valuation-based approaches and conjoint analysis-based approaches. OALY-based approaches combine estimates of life years gained and enhancement of health-related quality of life within a generic measure of health outcome that permits comparisons between disparate health programmes. ${ }^{4}$ If we proceed with the assumption that growth hormone therapy does not lengthen the life of children but rather enhances their health-related quality of life, the task is to identify the appropriate preference-based approach to measuring their health-related quality of life. The available scaling techniques for the task vary in their strength of foundation in economic theory, and health economists are still debating their relative merits. ${ }^{5}$ The time trade-off approach to measuring the health-related quality of life weight of a health state involves asking individuals to consider the relative amounts of time they would be willing to trade in order to survive in various health states. ${ }^{4}$ For example, children with growth hormone deficiency or their families could be asked how many years of their life expectancy with short stature they would be willing to give up to live their remaining life being 4 inches taller. The standard gamble approach to measuring the health-related quality of life weight of a health state uses hypothetical lotteries as a means of measuring individuals' preferences. These lotteries involve a choice between two alternatives: the certainty of one outcome and a gamble with two possible outcomes. For example, an individual might be asked to choose between the certainty of achieving an increased adult height of 2 inches and the gamble where there is a $50 \%$ chance of achieving a 4-inch height increase and a $50 \%$ chance of achieving no height increase at all. ${ }^{6}$ The probability of achieving a 4-inch height increase, as opposed to no height increase at all, is then varied until the individual shows no preference between the certain option and the gamble. An alternative preference-based approach to measuring the health-related quality of life weight of a health state is provided by multi-attribute utility measures, which are generic health-related quality of life instruments with pre-existing preference weights that can be attached to each permutation of responses. The available multi-attribute utility measures include the Quality of Well-Being Scale, ${ }^{7}$ EQ-5D, ${ }^{8}$ Health Utilities Index, ${ }^{9} 16 \mathrm{D},{ }^{10} 17 \mathrm{D}^{11}$ and SF-6D. ${ }^{12}$ The intuitive appeal of these instruments is that they allow each health state to be described using a simple health status classification, which can then be expressed as a health state preference value by reference to prescaled responses (usually obtained using the time trade-off or standard gamble approaches) from a relevant reference group. The Health Utilities Index is the most widely used multi-attribute utility measure in childhood.

\section{Perspective on the} papers by Ahmed et al

\section{(see page 110) and Kapoor et al (see page 147)}

An alternative preference-based approach that can be used to measure the benefits of growth hormone therapy in children is contingent valuation, also referred to in the literature as the willingness-to-pay approach. The technique was developed in environmental economics to value improvements to public goods, such as cleaner air. ${ }^{13}$ It is considered to provide values that reflect individuals' strength of preference for healthcare interventions. ${ }^{4}$ Willingness-to-pay questions can be classified in either openended or closed-ended form; the latter offers respondents an opportunity to accept or reject a series of price levels for the intervention being valued. Patient and population values may be obtained using a variety of survey techniques, including face-to-face interviews, telephone interviews and postal surveys.

An advantage of the contingent valuation approach is that, unlike OALY-based approaches, which focus on the health 
gain that can be attributed to an intervention, it permits respondents to take into account other factors, such as the value they attach to non-health outcomes or to the process of care. These other potential benefits of healthcare interventions may have clear importance in the context of growth hormone therapy in children. The study by Kapoor et $a l^{3}$ reminds us that children's compliance with growth hormone therapy may often be relatively poor. This may be the result of problems surrounding the doctorpatient relationship and the failure of the healthcare services to meet patients' expectations. ${ }^{14}$ The contingent valuation approach could, in theory, capture preferences for the way growth hormone therapy is conveyed and delivered, as well as its outcomes. One disadvantage of the contingent valuation approach is that people are often unwilling to place a value on health. Respondents may also give artificially high or low answers if they have an interest in prioritising one area of healthcare over another. Also, willingnessto-pay questions may generate responses that are associated with respondents' ability to pay. ${ }^{15}$ This can be controlled for in the analysis, but means that information about family income must also be elicited.

Conjoint analysis is a further preference-based technique that can be used to measure the benefits of growth hormone therapy in children. The technique was originally developed in the literature by mathematical psychologists and subsequently developed by market researchers, transport economists, environmental economists and, more recently, health economists. From its first application in healthcare in $1992,{ }^{16}$ the development of the technique by health economists has concentrated on one particular strand of the conjoint analysis approach, namely discrete choice experiments or discrete choice modelling. This is the preferred conjoint analysis approach of health economists because of its grounding in random utility theory ${ }^{17}$ as well as consumer choice theory..$^{18}$ In the health arena, the technique has been used as a means of eliciting individual and community preferences for interventions, models of care, or drug regimens. ${ }^{19}$ The technique is based on the premise that any "good"-in healthcare any intervention, drug therapy, treatment or model of care for example-can be described by its attributes (or characteristics) and that the extent to which an individual values a "good" depends on the level of these attributes. The attributes may describe the impact of the intervention or model of care on health outcomes, but may also describe non-health outcomes or the process by which the intervention or model of care is delivered..$^{20}$ As such, the technique shares many of the features of the contingent valuation approach, but has the additional feature of generating marginal rates of substitution between the attributes - that is, the degree to which respondents are willing to trade one attribute for another. Indeed, if cost is included as one of the study attributes, then marginal willingness-to-pay values can be inferred for changes in the levels of the remaining attributes. There are five identifiable stages in the design and analysis of conjoint analysis studies: (1) identifying the attributes to include in the study; (2) assigning levels to these attributes; (3) designing the orthogonal matrix of attributes and levels using design theory; (4) eliciting preferences for these scenarios; (5) analysing the responses. The technique has previously been used to evaluate parental attitudes about familyfocused prevention programmes ${ }^{21}$ and sexually transmitted infection vaccination programmes for their adolescent children. $^{22}$

In the context of growth hormone therapy in children, conjoint analysis can be used to assess the trade-offs that children and their families are willing to make in the delivery and outcomes of care-for example, the increased risk of adverse effects that they are willing to accept in order to achieve acceleration in linear growth. The study by Ahmed et al ${ }^{2}$ focuses on the preferences of children and their families for alternative attributes of growth hormone injection devices. The pharmaceutical industry has developed a plethora of growth hormone injection devices in recent years that differ in their attributes, such as their size, weight, method of subcutaneous injection, and associated support services provided. ${ }^{23}$ Ahmed et al identify 14 attributes that could influence preferences for devices in an outpatient clinic setting. The levels of bruising and pain experienced by patients and the ease with which the device can be held were not formally included in the authors' statistical model on the basis that they are subjective and difficult to quantify objectively. Previous discrete choice experiments, ${ }^{24}$ and preference elicitation studies more broadly, ${ }^{25}$ have overcome such quantification problems by providing study participants with extraneous information and the use of innovations such as pictorial representations and props.
A methodological issue that must be addressed by all studies that elicit preferences for growth hormone therapy in children is identifying the most appropriate respondents for the task. In their graded-pairs conjoint analysis, Ahmed et al ${ }^{2}$ elicited preferences from 56 children and their parents attending a paediatric endocrine clinic in Glasgow, Scotland. It is unclear from the information provided how much influence the children themselves had in the decision-making process. For the purpose of evaluating paediatric interventions, the psychometric literature presents compelling arguments in favour of eliciting preference data directly from children themselves. ${ }^{26}$ Each child is likely to have a unique perspective on, and valuation of, his or her healthcare experience or expectations, and may learn to conceal their true emotions from parents and carers. $^{26}$ Difficulties arise, however, when the child lacks the cognitive and linguistic skills necessary to complete preference-based measures. In this case, the preference elicitation task commonly falls on parents. Parent's views, however, are likely to be affected by their own experiences and expectations. ${ }^{26}$ Empirical evidence from the health-related quality of life literature suggests that parents are able to accurately rate the observable behaviours of their children, such as physical functioning and physical symptoms, but are less successful at identifying social or emotional impairments. For example, in a study of teenaged children born at extremely low birth weight, Saigal et $a^{27}$ measured health status by direct personal interviews independently with the children and their parents using the Health Utilities Index Mark II classification. Differences between the child-parent dyads were observed mainly in the cognition dimension, where children tended to describe themselves at a higher level of function than did their parents, and in the sensation dimension, where children identified more problems compared with parent reports. In the context of eliciting preferences for children's growth hormone injection devices, therefore, it is conceivable that parental views do not accurately reflect some aspects of the childhood experience.

A further consideration for researchers eliciting preferences for growth hormone therapy in children is whether the measurement approach is methodologically sound-that is, whether it satisfies a number of psychometric properties. ${ }^{5}$ The criterion of practicality implies that the measurement approach should be acceptable to the respondent whose preferences 
are being measured. Consideration should therefore be given to the length of time taken to administer the instrument, as well as its feasibility, cost and the quality of the data. A useful test of external validity is to compare the stated preference results with observed behaviour in practice. In the study by Ahmed et $a l^{2}$ there was a high degree of correlation between the preferred devices predicted by the statistical model and the actual devices used by children. This suggests an encouraging level of discourse between families and healthcare staff about the appropriate devices for individual cases. Furthermore, some recent discrete choice experiments have involved follow-up debriefing with respondents about their experience of completing the research instruments. Quantitative and qualitative debriefing methods can provide insight into the respondents' understanding of the experiment, the validity of the choice sets, and their cognitive processes when selecting between choice sets. ${ }^{28}$

How should the results of preference elicitation studies be integrated into clinical decision-making? Ahmed et al argue that, in the context of growth hormone therapy in children, their conjoint analysis technique can be readily adapted to the setting of the paediatric endocrine clinic. It is argued that the technique can be used to generate individual preferences, which in turn could personalise the injection devices that children receive. ${ }^{2}$ However, would this process of eliciting patient preferences in order to inform the delivery of growth hormone therapy injection devices be a cost-effective use of scarce healthcare resources? There is little evidence at present to suggest that clinical outcomes differ for the various injection devices. ${ }^{29}$ Although there may be patient and parent preferences for some of the devices, an individualised approach to preference elicitation in a clinic setting is likely to be both burdensome and expensive, with little to show in terms of clinical benefits. Implementing an individualised approach would require healthcare professionals to be trained in the minutiae of conjoint analysis methodology, as well as $30 \mathrm{~min}$ interviews for each of the approximately 2000 children in Britain receiving growth hormone therapy. ${ }^{30}$ The opportunity costs of implementation could be substantial and result in foregoing of the delivery of costeffective alternative therapies. A useful way forward may be to use evidence from preference elicitation studies to inform guidelines or recommendations at a macro level within publicly funded healthcare systems. This may translate into national or clinic-level guidelines in favour of particular devices or prescribing policies on the basis of patient or community preferences. Such an approach would circumvent the need for interviews with each patient with growth hormone deficiency, Turner syndrome, idiopathic short stature, etc, presenting for treatment.

More broadly, evidence from preference elicitation studies could be channelled into a decision-analytical framework that combines the valuable aspects of evidencebased, cost-effective and preference-driven medicine. ${ }^{31}$ Interpreting preference data in isolation cannot identify the most efficient allocation of finite healthcare resources. Rather, decision-makers are increasingly interested in viewing evidence on incremental costs, incremental health gains, and patient and community preferences attributable to healthcare interventions in synthesis. In the context of growth hormone therapy in children, this would require an assessment and synthesis of different strands of evidence, including the cost of interventions and systems, their impact on short- and long-term outcomes, as well as patient and community preferences. An explicit decision-analytical framework that combines all relevant strands of evidence could be used to inform a rational and efficient allocation of finite resources in this area.

\section{Competing interests: None.}

Arch Dis Child 2008;93:95-97. doi:10.1136/adc.2007.129650

\section{REFERENCES}

1. Vance $\mathbf{M L}$, Mauras N. Growth hormone therapy in adults and children. N Engl J Med 1999;341:1206-16

2. Ahmed SF, Blamires C, Smith WA. Facilitating and understanding the family's choice of injection devise for growth hormone therapy by using conjoint analysis. Arch Dis Child 2008;93:110-4.

3. Kapoor RR, Burke S, Sparrow S, et al. Monitoring of concordance in growth hormone therapy. Arch Dis Child 2008;93:147-8.

4. Drummond MF, O'Brien B, Stoddart GL, et al. Methods for the economic evaluation of health care programmes. 2nd edn. Oxford: Oxford University Press, 1997.

5. Brazier J, Deverill M, Green C, et al. A review of the use of health status measures in economic evaluation. Health Technol Assess 1999;3:1-164

6. Pliskin JS. Towards better decision making in growth hormone therapy. Horm Res 1995;51:30-5.

7. Kaplan RM, Bush JW, Berry CC. Health status: types of validity and the index of well-being. Health Serv Res 1976;11:478-507.

8. Euro0ol Group. Euro0ol: a new facility for the measurement of health-related quality of life. Health Pol 1990;16:199-208.

9. Torrance GW, Furlong W, Feeny D, et al. Multiattribute preference functions: Health Utilities Index. PharmacoEconomics 1995; 7:503-20.
10. Apajasalo M, Sintonen C, Holmberg C, et al. Quality of life in early adolescence: a sixteen-dimensional health-related measure (16D). Qual Life Res 1996;5:205-11.

11. Apajasalo M, Rautonen J, Holmberg C, et al. Quality of life in pre-adolescence: a seventeen-dimensional health-related measure (17D). Qual Life Res 1996;5:532-8.

12. Brazier J, Roberts J, Deverill M. The estimation of a preference-based measure of health from the SF-36. $J$ Health Econ 2002;21:271-92.

13. In: Cummings RG, Brookshire DS, Schulze WD, eds Valuing environmental goods. Totowa, NJ: Rowman and Allanheld, 1986.

14. Hindmarsh PC, Brook CDG. Compliance with growth hormone treatment: is it a problem? Horm Res 1999:61:104-8.

15. O'Brien B, Viramontes JL. Willingness to pay: a valid and reliable measure of health state preference. Med Decis Making 1994;14:289-97.

16. Maas A, Stalpers L. Assessing utilities by means of conjoint measurement: an application in medical decision analysis. Med Decis Making 1992;12:28897.

17. Manski CF. The structure of random utility models. Theor Decis 1977;8:254.

18. Freeman AM. The measurement of environmental and resource values: theory and methods. 3rd edn. Washington: Resources for the Future, 1993.

19. Ryan M, Farrar S. Using conjoint analysis to elicit preferences for health care. BMJ 2000;320:1530-3.

20. Ryan M. Using conjoint analysis to take account of patient preferences and go beyond health outcomes: an application to in-vitro fertilisation. Soc Sci Med 1999;48:535-46.

21. Spoth R, Redmond C. Identifying program preferences through conjoint analysis: illustrative results from a parent sample. Am J Health Promot 1993;8:124-33.

22. Zimet GD, Mays RM, Sturm LA, et al. Parental attitudes about sexually transmitted infection vaccination for their adolescent children. Arch Pediatr Adolesc Med 2005;159:132-7.

23. Fidotti E. A history of growth hormone injection devices. J Pediatr Endocrinol Metab 2001;14:497501.

24. Gidman W, Elliott R, Payne K, et al. A comparison of parents and paediatric anesthesiologists' preferences for attributes of child day case surgery: a discrete choice experiment. Paediatr Anaesth 2007;17:104352.

25. Petrou S. Methodological issues raised by preference-based approaches to measuring the health status of children. Health Econ 2003;12:697-702.

26. Eiser C, Morse R. Quality-of-life measures in chronic diseases of childhood. Health Technol Assess 2001;5:1-156.

27. Saigal S, Rosenbaum PL, Feeny D, et al. Parental perspectives of the health status and health-related quality of life of teen-aged children who were extremely low birth weight and term controls. Pediatrics 2000;105:569-74.

28. Miguel FS, Ryan M, Amaya-Amaya M. 'Irrational' stated preferences: a quantitative and qualitative investigation. Health Econ 2005;14:307-22.

29. Wilson TA, Rose SR, Cohen P, et al. Update of guidelines for the use of growth hormone in children: the Lawson Wilkins Pediatric Endocrinology Society Drug and Therapeutics Committee. J Pediatr 2003; 143:415-21.

30. Bryant J, Cave C, Mihaylova B, et al. Clinical effectiveness and cost-effectiveness of growth hormone in children: a systematic review and economic evaluation. Health Technol Assess 2002;6:1-175.

31. Dowie J. 'Evidence-based', 'cost-effective' and 'preference-driven' medicine: decision analysis based medical decision making is the pre-requisite. J Health Serv Res Policy 1996;1:104-13. 